\title{
Logically constrained optimal power flow: Solver-based mixed-integer nonlinear programming model
}

\author{
Mahdi Pourakbari-Kasmaei*, José Roberto Sanches Mantovani \\ Department of Electrical Engineering, State University of Sao Paulo, Itha Solteira, Brazil
}

\section{A R T I C L E I N F O}

\section{Keywords:}

FACTS devices

Logical constraint

Mixed-integer nonlinear programming

Optimal power flow

Solver-based model

Non-smooth terms

\begin{abstract}
A B S T R A C T
There is increasing evidence of the shortage of solver-based models for solving logically-constrained AC optimal power flow problem (LCOPF). Although in the literature the heuristic-based models have been widely used to handle the LCOPF problems with logical terms such as conditional statements, logical-and, logical-or, etc., their requirement of several trials and adjustments plagues finding a trustworthy solution. On the other hand, a welldefined solver-based model is of much interest in practice, due to rapidity and precision in finding an optimal solution. To remedy this shortcoming, in this paper we provide a solver-friendly procedure to recast the logical constraints to solver-based mixed-integer nonlinear programming (MINLP) terms. We specifically investigate the recasting of logical constraints into the terms of the objective function, so it facilitates the pre-solving and probing techniques of commercial solvers and consequently results in a higher computational efficiency. By applying this recast method to the problem, two sub-power- and sub-function-based MINLP models, namely SPMINLP and SF-MINLP, respectively, are proposed. Results not only show the superiority of the proposed models in finding a better optimal solution, compared to the existing approaches in the literature, but also the effectiveness and computational tractability in solving large-scale power systems under different configurations.
\end{abstract}

\section{Introduction}

Logical constraints, which are one particular kind of discrete or numerical constraints such as logical-and, logical-or, negation, and conditional statements, are considered as the nature of most practical optimization problems, and the practical power systems are no exception. Although the logical constraints exist in most decision-making problems of power systems, due to disjoint functioning regions of generating units, more often than not, for the sake of simplicity and computational tractability, these constraints are neglected. This may facilitate finding an optimal solution, however, on the other hand, an accurate model should embody all operational constraints, otherwise, it may lead to a solution with an unsatisfactory outcome. Thus, an appropriate model or tradeoff, between model accuracy and computational efficiency, should be investigated to counteract the aforementioned drawback.

The AC optimal power flow (ACOPF) problem, even in the theoretical studies, is a highly nonlinear problem, due to active and reactive power flow constraints [1], and considering logical constraints makes it even a more complex and highly nonconvex-nonlinear problem. On the other hand, to have a more practical model, the valve-point effect should be considered [2,3], and this exponentially increases the degree of nonlinearity of the problem. Moreover, considering shunt VAr compensator and more specifically thyristor controlled series capacitor (TCSC) and thyristor controlled phase shifter (TCPS) play a crucial role in practical power system operation and planning problems, by improving the efficiency, voltage fluctuations, and loadability. In planning-based problems, the optimal siting and sizing of FACTS devices are taken into account $[4,5]$, while in operating-based problems, the adjustment of these devices, which have already been optimally placed and sized, is considered [6-8]. Incorporating such devices with highnonlinearity characteristics besides the integer variables of logical constraints results in a complex mixed-integer nonlinear programming problem. The price to be paid for considering the logical constraints and the flexible AC transmission systems (FACTS) is a dramatic increase in the degree of computational complexity, which if handled without care may lead to intractability. This is one of the main motivations of widely using heuristic-based approaches to solve the practical OPF-based problems [9-12]. These approaches may work well in finding an optimal solution for specific systems or models; however, finding an acceptable solution for other systems and models, especially when logical constraints are taken into account, may require major modifications and adjustments. On the other hand, the most successful approaches, among others, to solve OPF problems such as interior point method

\footnotetext{
* Corresponding author at: Avenida Brasil, 56, Bairro: Centro, 15385-000 Ilha Solteira, SP, Brazil.

E-mail address: mahdi.pourakbari@ieee.org (M. Pourakbari-Kasmaei).
} 


\section{Nomenclature \\ (a) Indices

$\begin{array}{ll}i, j & \text { bus indices } \\ k & \text { index for disjoint operating zones } \\ i j & \text { index for the transmission line or device between bus } i \text { and } \\ & j \\ d & \text { index for direct power flow } \\ r & \text { index for reverse power flow }\end{array}$

(b) Sets

$\begin{array}{ll}\Omega_{b} & \text { set of buses, }\left\{1,2, \ldots, N_{b}\right\} \\ \Omega_{g} & \text { set of generating units, }\left\{1,2, \ldots, N_{g}\right\}, \Omega_{g} \subseteq \Omega_{b} \\ \Omega_{l} & \text { set of transmission elements, }\left\{1,2, \ldots, N_{l}\right\}\end{array}$

\section{(c) Variables and Functions}

\begin{tabular}{|c|c|}
\hline$F_{i}(\cdot)$ & fuel cost function of unit $i$ \\
\hline$f l_{i j}$ & power flow at branch $i j$ \\
\hline$n_{i}$ & $\begin{array}{l}\text { nonnegative integer decision making variable for shunt } \\
\text { VAR compensator at bus } i\end{array}$ \\
\hline$n_{i j}$ & $\begin{array}{l}\text { nonnegative integer decision making variable for LTCT at } \\
\text { branch } i j\end{array}$ \\
\hline$P_{g_{i}}$ & active power generation of unit $i$ \\
\hline$P_{i k}$ & $\begin{array}{l}\text { active power corresponding to the operating zone } k \text { of unit } \\
i \text {; used in MINLP models }\end{array}$ \\
\hline$p_{i j}^{d} / p_{i j}^{r}$ & $\begin{array}{l}\text { direct/reverse active power between bus } i \text { and bus } j \text { of } \\
\text { branch } i j\end{array}$ \\
\hline$q_{i j}^{d} / q_{i j}^{r}$ & $\begin{array}{l}\text { direct/reverse reactive power between bus } i \text { and bus } j \text { of } \\
\text { branch } i j\end{array}$ \\
\hline$Q_{c_{i}}$ & shunt VAR compensation of bus $i$ \\
\hline$Q_{g_{i}}$ & reactive power generation of unit $i$ \\
\hline$t p_{i j}$ & transformer tap of branch $i j$ \\
\hline$u_{i k}$ & $\begin{array}{l}\text { binary decision making variables of unit } i \text { and operating } \\
\text { zone } k\end{array}$ \\
\hline$v_{i}$ & voltage magnitude at bus $i$ \\
\hline$x_{i j}^{c}$ & reactance of TCSC at branch $i j$ \\
\hline$\delta_{i}$ & voltage angle of bus $i$ \\
\hline
\end{tabular}

(IPM) [13], primal-dual IPM [14], predictor corrector IPM (PCIPM) [15], decomposed PCIPM [16], trust region and IPM [17], which are known as the standard methods to solve OPF problems, may properly find an optimal solution for the conventional OPF problems, but when applied to OPF problems with logical constraints and FACTS devices (with discrete decision variables), the reliability of them should be seriously questioned [18]. This shows the exigency of proposing a trustworthy model for logical constrained problems.

Until now, to the best of our knowledge, for the logically constrained ACOPF (LCOPF) problems (with or without considering VAr compensators) there is no solver-based model, although, for the logically constrained economic dispatch (LCED) problem, which is a simplified OPF problem, several solver-based models have been proposed. First, in [19], a mixed-integer quadratic programming (MIQP) model has been proposed, and later, in [20] and [21], the authors have developed the MIQP model by proposing a big-M based MIQP (M-MIQP) model and an unambiguous distance-based MIQP (UDB-MIQP) model, respectively. These models could obtain the global optimal solution of ED problems; however, their incapability in dealing with non-smooth and nonlinear terms is still an undeniable shortcoming that prevents them to be applied to LC-ACOPF problems. In order to consider the nonlinear terms such as transmission losses, first, in [22], and later, in [23], a novel transformation has been introduced. Such transformation $\theta_{i j} \quad$ voltage angle difference between bus $i$ and $j, \theta_{i j}=\delta_{i}-\delta_{j}$.

$\varphi_{i j} \quad$ phase shift angle of TCPS at branch $i j$

\section{(d) Constants}

\begin{tabular}{|c|c|}
\hline$a_{i}, b_{i}, c_{i}$ & cost coefficients of unit $i$ \\
\hline$b_{i j}^{c h}$ & charging susceptance of branch $i j$ \\
\hline$b_{i}^{\text {sh }}$ & shunt susceptance of bus $i(\widetilde{)})$ \\
\hline$b_{i j}$ & susceptance of branch $i j(\widetilde{)})$ \\
\hline$e_{i} f_{i}$ & valve-point cost coefficients of unit $i$ \\
\hline$\overline{f l}_{i j}$ & maximum power flow of branch $i j$ \\
\hline$g_{i j}$ & conductance of branch $i j(\Omega)$ \\
\hline$g_{i}^{s h}$ & shunt conductance of bus $i(\Omega)$ \\
\hline$P_{D_{i}}$ & active power demand at bus $i$ \\
\hline$\underline{P_{g_{i}}}, \bar{P}_{g_{i}}$ & $\begin{array}{l}\text { minimum and maximum active power generation limits of } \\
\text { unit } i \text {, respectively }\end{array}$ \\
\hline$P_{g_{i k}}, \bar{P}_{g_{i k}}$ & $\begin{array}{l}\text { minimum and maximum active power limits correspond to } \\
\text { operating zone } k \text { of unit } i \text {, respectively }\end{array}$ \\
\hline$\underline{P_{i k}}, \bar{P}_{i k}$ & $\begin{array}{l}\text { minimum and maximum active power limits correspond to } \\
\text { operating zone } k \text { of unit } i \text {, respectively; used in MINLP } \\
\text { models }\end{array}$ \\
\hline$Q_{D_{i}}$ & reactive power demand at bus $i$ \\
\hline$Q_{C_{i}}, \bar{Q}_{C_{i}}$ & $\begin{array}{l}\text { upper and lower limits of shunt VAR compensator at bus } i \text {, } \\
\text { respectively }\end{array}$ \\
\hline$\underline{Q_{g_{i}}}, \bar{Q}_{g_{i}}$ & $\begin{array}{l}\text { minimum and maximum reactive power generation limits } \\
\text { of unit } i \text {, respectively }\end{array}$ \\
\hline$r_{i j}$ & resistance of branch $i j(\Omega)$ \\
\hline$\underline{t p}_{i j}, \overline{t p}_{j i}$ & $\begin{array}{l}\text { minimum and maximum limits of transformer tap of } \\
\text { branch } i j \text {, respectively }\end{array}$ \\
\hline$\underline{v}_{i}, \bar{v}_{i}$ & $\begin{array}{l}\text { minimum and maximum voltage magnitude limits of bus } i \text {, } \\
\text { respectively }\end{array}$ \\
\hline$x_{i j}$ & reactance of branch $i j(\Omega)$ \\
\hline$x_{i j}^{c}, \bar{x}_{i j}^{c}$ & minimum and maximum reactance of TCSC at branch $i j$ \\
\hline$\overline{z_{i}}$ & number of operating zones for unit $\underline{i}$ \\
\hline$\tau_{i}$ & predefined step size for shunt VAR compensator at bus $i$ \\
\hline$\tau_{i j}$ & predefined step size for LTCT at branch $i j$ \\
\hline$\underline{\varphi_{i j}}, \bar{\varphi}_{i j}$ & $\begin{array}{l}\text { minimum and maximum limits of phase shift angle of } \\
\text { TCPS at branch } i j\end{array}$ \\
\hline
\end{tabular}

may cause significant difficulties for the commercial solvers since (a) it results in non-constant upper and lower limits, and (b) the operation of a unit in only one operating zone is guaranteed by forcing the product of two continuous variables, correspond with two different operating zones of that unit, equals to zero, which is a very-hard equality constraint and causes severe difficulties for commercial nonlinear solvers. Therefore, to deal with this problem, in [22], a semidefinite approach, and in [23], a decomposition technique has been used. Although the aforementioned models are not capable of solving practical-constrained models (either complex ED or OPF problems), they have brought new insights into this area of research by showing the importance of solverbased models. Even in some existing linear models for ACOPF problems, [24] and [25], due to the complexity of linearization that highly depends on the approximation techniques, the logical constraints have been neglected. Therefore, the main motivations of proposing the solver-based MINLP models that may fill the existing gap in this area of research can be summarized as (a) the popularity and efficient outcomes of solver-based models in other areas, and (b) the lack of an efficient solver-based model for LCOPF-based problems. Accordingly, the contributions of this paper are threefold:

(1) A transformation of logical characteristics to mixed-integer nonlinear terms by recasting them to the objective function as: 
(a) the sub-objective function terms; this results in a sub-functionbased LCOPF (SF-LCOPF) in which each sub-function corresponds with an operating zone.

(b) the sub-generating regions terms; this results in a sub-powerbased LCOPF (SP-LCOPF) in which each sub-power corresponds with an operating region.

(2) The mathematical formulations of the proposed models, unlike the other practical models in the literature, are adapted to the presolving and probing techniques of commercial solvers. This results in a reduction of the model complexity by reducing the size of the problem via eliminating unnecessary variables and constraints in the pre-solve step and improving the formulation via pre-processing and probing techniques, which rely mainly on the model's simplicity. During preprocessing, the infeasibility and redundancy, bounds improvement, and rounding (for MIP) are considered, while in probing, fixing the variables, coefficients improvement and the logical implications are taken into account [21]. Another advantage of these solver-friendly models is that they can be easily implemented in modeling language-based systems such as AMPL [26], GAMS [27], etc.

(3) Testing the flexibility of the proposed logically-constrained models for dealing with cost or loss minimization problems under different topologies such as (a) power systems operation considering prohibited operating zones, (b) power systems operation considering nonsmooth terms, such as valve-point effect, and (c) power systems operation considering flexible AC transmission system (FACTS), such as thyristor controlled series capacitor (TCSC) and thyristor controlled phase shifter (TCPS).

To evaluate and validate the proposed MINLP models and demonstrate their effectiveness compared to the existing approaches in the literature, the commonly used IEEE 30-bus system is considered in detail, while in order to show their performance in solving large-scale and very large-scale power systems the IEEE 118-bus and Polish 2383bus test systems are investigated, respectively.

The rest of this paper is organized as follows. Section 2 contains the mathematical formulation of the proposed MINLP models. Case studies and results are presented in Section 3. Section 4 contains the concluding remarks and the prospects for future works.

\section{Mixed integer nonlinear programming model for logically constrained OPF}

The mathematical model of the logically constrained OPF (LCOPF) is presented, first, and then its equivalent mixed-integer nonlinear programming models are considered in detail.

\subsection{Mathematical formulation of LCOPF}

In order to show the wide application of our proposed recast technique, three mathematical formulations correspond with three different configurations are presented, such as (a) OPF with prohibited operating zones, named as logically-constrained OPF (LCOPF), (b) LCOPF considering shunt VAR compensator and load-tap-changing transformer, and (c) LCOPF with TCSC and TCPS.

\subsubsection{F1: Logically constrained OPF (LCOPF)}

The OPF problem with considering disjoint operating zones, as logical constraints, is formulated as follows.

$\min \sum_{i \in \Omega_{g}} F_{i}\left(P_{g_{i}}\right)$

s.t.

$P_{g_{i}}-P_{D_{i}}-g_{i}^{s h} v_{i}^{2}-\sum_{i j \in \Omega_{l}} p_{i j}^{d}-\sum_{j i \in \Omega_{l}} p_{j i}^{r}=0, i \in \Omega_{b}$
$Q_{g_{i}}-Q_{D_{i}}+b_{i}^{s h} v_{i}^{2}-\sum_{i j \in \Omega_{l}} q_{i j}^{d}-\sum_{j i \in \Omega_{l}} q_{j i}^{r}=0, i \in \Omega_{b}$

$\left\{\begin{array}{l}\underline{P}_{g_{i}}=\underline{P}_{g_{i 1}} \leqslant P_{g_{i}} \leqslant \bar{P}_{g_{i 1}}, \text { or } \\ \underline{P}_{g_{i \mathrm{k}}} \leqslant P_{g_{i}} \leqslant \bar{P}_{g_{i \mathrm{k}}}, \forall \quad \leqslant k \leqslant\left(z_{i}-1\right), \text { or } \\ \underline{P}_{g_{i z_{i}}} \leqslant P_{g_{i}} \leqslant \bar{P}_{g_{i z_{i}}}=\bar{P}_{g_{i}}\end{array}\right.$

$\underline{Q_{g_{i}}} \leqslant Q_{g_{i}} \leqslant \bar{Q}_{g_{i}}, i \in \Omega_{g}$

$\underline{v}_{i} \leqslant v_{i} \leqslant \bar{v}_{i}, i \in \Omega_{b}$

$\left|f l_{i j}(v, \theta, t p)\right| \leqslant \overline{f l}_{i j}, i j \in \Omega_{l}$

where $F_{i}(\cdot)$ is mostly approximated by a quadratic function, as (8).

$F_{i}\left(P_{g_{i}}\right)=a_{i} P_{g_{i}}^{2}+b_{i} P_{g_{i}}+c_{i}$

However, in practice, multiple valves result in the ripples and therefore considering the valve-point effects in cost function is inevitable [28]. The valve-point effect is modeled as a rectified sinusoidal term as (9)

$F_{i}\left(P_{g_{i}}\right)=a_{i}\left(P_{g_{i}}\right)^{2}+b_{i} P_{g_{i}}+c_{i}+\left|e_{i} \sin \left(f_{i}\left(\underline{P}_{g_{i}}-P_{g_{i}}\right)\right)\right|$

The active and reactive equality constraints are represented in (2) and (3) $[29,30]$, respectively.

The set of equations in (4) stands for disjoint operating zones [31]; this set brings the concept of logical constraints to the OPF problems. The limits of reactive power generations and bus voltages are represented by (5) and (6), respectively.

In (7), the power flows of branches, $f_{i j}$, can be defined by (10) or in some texts only the active power flow of lines are taken into account [32].

$f l_{i j / j i}=\sqrt{\left(p_{i j / j i}^{d / r}\right)^{2}+\left(q_{i j / j i}^{d / r}\right)^{2}}$

\subsubsection{F2: LCOPF with shunt VAR compensator and load-tap-changing transformer (LTCT)}

In this model, the load-tap-changing transformer (LTCT) and shunt VAR compensator are considered in LCOPF. By incorporating the LTCT devices in a power system, as Fig. 1, the direct and reverse flows of active and reactive powers in transmission lines are defined as follows, (11)-(14) [33].

$p_{i j}^{d}=\left(t p_{i j} v_{i}\right)^{2} g_{i j}-\left(t p_{i j} v_{i}\right) v_{j}\left[g_{i j} \cos \left(\theta_{i j}\right)+b_{i j} \sin \left(\theta_{i j}\right)\right]$

$p_{i j}^{r}=v_{j}^{2} g_{i j}-\left(t p_{i j} v_{i}\right) v_{j}\left[g_{i j} \cos \left(\theta_{i j}\right)-b_{i j} \sin \left(\theta_{i j}\right)\right]$

$q_{i j}^{d}=-\left(t p_{i j} v_{i}\right)^{2}\left(b_{i j}+\frac{b_{i j}^{c h}}{2}\right)-v_{i} v_{j}\left[g_{i j} \sin \left(\theta_{i j}\right)-b_{i j} \cos \left(\theta_{i j}\right)\right]$

$q_{i j}^{r}=-v_{j}^{2}\left(b_{i j}+\frac{b_{i j}^{c h}}{2}\right)+\left(t p_{i j} v_{i}\right) v_{j}\left[g_{i j} \sin \left(\theta_{i j}\right)+b_{i j} \cos \left(\theta_{i j}\right)\right]$

where each tap must satisfy its lower and upper limits as (15).

$\underline{t}_{i j} \leqslant t p_{i j} \leqslant \overline{t p}_{i j}$

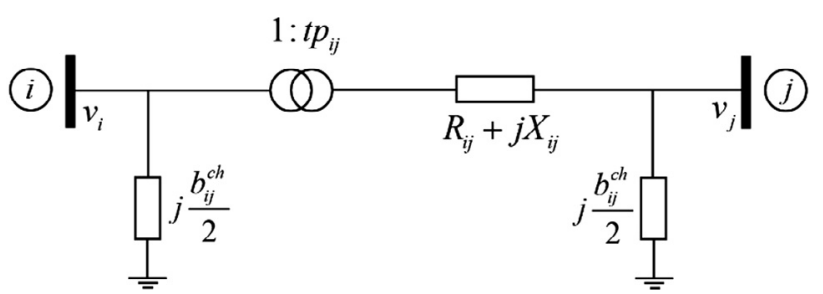

Fig. 1. A transmission line model at the presence of LTCT. 
And to incorporate the effects of shunt VAR compensator, as Fig. 2, the reactive equality constraint (3) is modified as (16).

$Q_{g_{i}}+Q_{C_{i}}-Q_{D_{i}}+b_{i}^{s h} v_{i}^{2}-\sum_{i j \in \Omega_{l}} q_{i j}^{d}-\sum_{j i \in \Omega_{l}} q_{j i}^{r}=0, i \in \Omega_{b}$

where each shunt VAR compensator has its limitations [34,35], as (17).

$Q_{C_{i}} \leqslant Q_{C_{i}} \leqslant \bar{Q}_{C_{i}}$

On the other hand, in practice, the LTCTs and shunt VAR compensators are discrete controllers with predefined step sizes [34]. These devices can be modeled by using integer decision maker variables, as follows.

$t p_{i j}=\underline{t p}_{i j}+n_{i j} \tau_{i j}, \quad \forall n_{i j} \in \mathbb{Z}_{\geqslant 0}$

$Q_{C_{i}}=Q_{C_{i}}+n_{i} \tau_{i}, \quad \forall n_{i} \in \mathbb{Z}_{\geqslant 0}$

\subsubsection{F3: $L C O P F$ with TCSC and TCPS}

In this model, the effects of specific FACTS devices such as the thyristor controlled series capacitor (TCSC) and thyristor controlled phase shifter (TCPS) are considered.

By considering TCPS, which can be modeled by a phase shifter transformer with control variable $\varphi_{i j}$, as Fig. 3 , the direct and indirect active and reactive powers are defined as follows, (20)-(23), respectively.

$p_{i j}^{d}=\frac{v_{i}^{2} g_{i j}}{\cos ^{2} \varphi_{i j}}-\frac{v_{i} v_{j}}{\cos \varphi_{i j}}\left[g_{i j} \cos \left(\theta_{i j}+\varphi_{i j}\right)+b_{i j} \sin \left(\theta_{i j}+\varphi_{i j}\right)\right]$

$p_{i j}^{r}=v_{j}^{2} g_{i j}-\frac{v_{i} v_{j}}{\cos \varphi_{i j}}\left[g_{i j} \cos \left(\theta_{i j}+\varphi_{i j}\right)-b_{i j} \sin \left(\theta_{i j}+\varphi_{i j}\right)\right]$

$q_{i j}^{d}=-\frac{v_{i}^{2}}{\cos ^{2} \varphi_{i j}}\left(b_{i j}+\frac{b_{i j}^{c h}}{2}\right)-\frac{v_{i} v_{j}}{\cos \varphi_{i j}}\left[g_{i j} \sin \left(\theta_{i j}+\varphi_{i j}\right)-b_{i j} \cos \left(\theta_{i j}+\varphi_{i j}\right)\right]$

$q_{i j}^{r}=-v_{j}^{2}\left(b_{i j}+\frac{b_{i j}^{c h}}{2}\right)+\frac{v_{i} v_{j}}{\cos \varphi_{i j}}\left[g_{i j} \sin \left(\theta_{i j}+\varphi_{i j}\right)+b_{i j} \cos \left(\theta_{i j}+\varphi_{i j}\right)\right]$

And by taking into account the TCSC with the control variable $x_{i j}^{c}$, as Fig. 4, the direct and indirect active and reactive power flows are defined as follows, (24)-(27), respectively, while taking into account (28) and (29) [36].

$p_{i j}^{d}=v_{i}^{2} g_{i j}-v_{i} v_{j}\left[g_{i j} \cos \left(\theta_{i j}\right)+b_{i j} \sin \left(\theta_{i j}\right)\right]$

$p_{i j}^{r}=v_{j}^{2} g_{i j}-v_{i} v_{j}\left[g_{i j} \cos \left(\theta_{i j}\right)-b_{i j} \sin \left(\theta_{i j}\right)\right]$

$q_{i j}^{d}=-v_{i}^{2}\left(b_{i j}+\frac{b_{i j}^{c h}}{2}\right)-v_{i} v_{j}\left[g_{i j} \sin \left(\theta_{i j}\right)-b_{i j} \cos \left(\theta_{i j}\right)\right]$

$q_{i j}^{r}=-v_{j}^{2}\left(b_{i j}+\frac{b_{i j}^{c h}}{2}\right)+v_{i} v_{j}\left[g_{i j} \sin \left(\theta_{i j}\right)+b_{i j} \cos \left(\theta_{i j}\right)\right]$

where the conductance, $g_{i j}$, and susceptance, $b_{i j}$, of lines with TCSC are calculated as follows.

$g_{i j}=\frac{r_{i j}}{r_{i j}^{2}+\left(x_{i j}-x_{i j}^{c}\right)^{2}}$

$b_{i j}=-\frac{x_{i j}-x_{i j}^{c}}{r_{i j}^{2}+\left(x_{i j}-x_{i j}^{c}\right)^{2}}$

Therefore by considering the aforementioned modifications as well as taking into account the two following constraints, (30) and (31), the LCOPF model with FACTS devices, TCSC and TCPS, is obtained.

$\underline{x}_{i j}^{c} \leqslant x_{i j}^{c} \leqslant \bar{x}_{i j}^{c}, i j \in \Omega_{l}$ $\underline{\varphi}_{i j} \leqslant \varphi_{i j} \leqslant \bar{\varphi}_{i j}, i j \in \Omega_{l}$

\subsection{Mixed integer nonlinear programming models for $L C O P F$}

Since the existing commercial nonlinear solvers are not capable of solving the logical constrained problems, the logical constraints must be recast to solver-friendly terms, and it becomes possible via mixed-integer reformulations. On the other hand, because of the highly nonlinear and nonconvex nature of LCOPF problems, the existing solverbased models for ED problems are not suitable, as these models bring difficulties in MINLP solvers' process. In such models, increasing the number of variables, by assigning the decision-making variables to the upper and lower limits of the corresponding constraints, is not the only hurdle that makes it inutile for LCOPF-based problems; however, the potential shortage of the existing mixed-integer nonlinear solvers is another obstacle. The main idea of our proposed mixed-integer nonlinear programming (MINLP) models comes from this fact that an MINLP solver shows higher performance when the decision-making variables are included in the objective function. Therefore, to address the aforementioned shortcomings, in this paper, two MINLP models for LCOPF problems are proposed where the logical constraints are recast to the term of objective function. These models are explained in detail as follows.

\subsubsection{Sub-power-based LCOPF}

Fig. 5 demonstrates the input-output curve of unit $i$ with considering valve-point effects and POZs where the dashed and solid blue curves stand for the quadratic cost function (8), and the cost function considering the valve-point effect (9), respectively. The red hachured regions represent the POZs of unit $i$.

Constraint (4) demonstrates that each unit can generate in only one operating zone. As it can be seen in Fig. 5, in order to obtain the MINLP model, first, the unit's input-output curve is divided into several subpower regions $\left(P_{i 1}, P_{i 2}\right.$, and $\left.P_{i 3}\right)$ corresponding to the operating zones. The boundaries of each sub-power are defined as (32).

$\underline{P}_{i \mathrm{k}} \leqslant P_{i \mathrm{k}} \leqslant \overline{P_{\mathrm{k}}} ; \forall i \in \Omega_{\mathrm{g}}, k \in\left\{1, \ldots, Z_{i}\right\}$

Then, to select one sub-power region, the binary decision-making variables $u_{i k}$ are assigned to these regions as (33) and (34). Constraint (34) guarantees that only one of the decision-making variables is set to one, and consequently, only one of the sub-power regions is selected to calculate the output power of unit $i$ in (33).

$P_{g_{i}}=P_{i 1} u_{i 1}+P_{i 2} u_{i 2}+\cdots+P_{i z_{i}-1} u_{i z_{i}-1}+P_{i z_{i}} u_{i z_{i}}=\sum_{k=1}^{z_{i}} P_{i k} u_{i k}$

$\sum_{k=1}^{z_{i}} u_{i k}=1, \quad \forall u_{i k} \in\{0,1\}$

By putting (33) in the objective function, (8) or (9), the sub-powerbased MINLP (SP-MINLP) model is obtained.

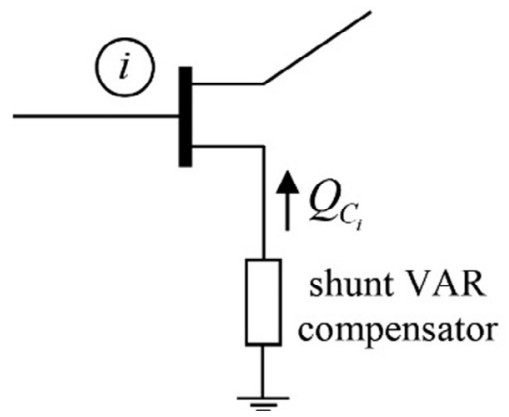

Fig. 2. Shunt VAR compensator connected to a bus. 


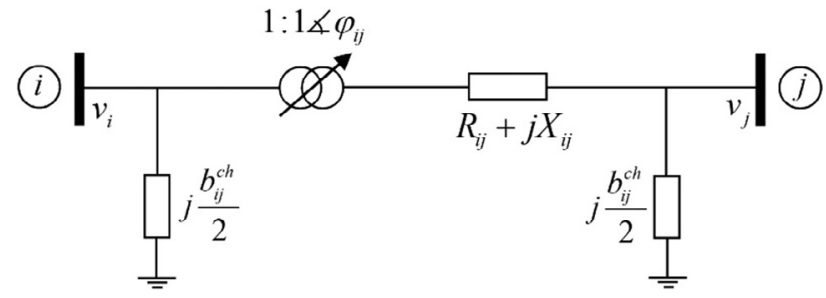

Fig. 3. A transmission line model at the presence of TCPS.

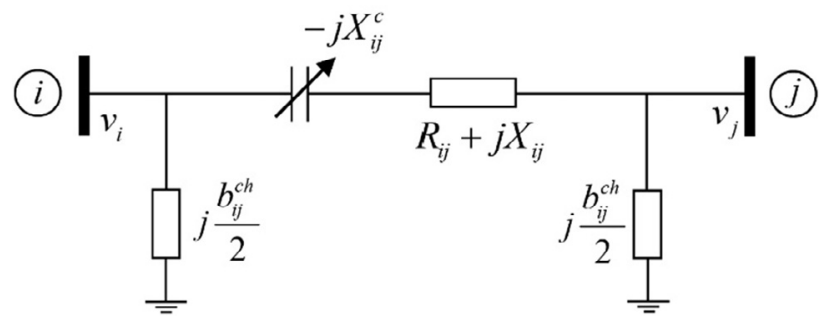

Fig. 4. A transmission line model at the presence of TCSC.

$$
\begin{aligned}
F_{i}\left(P_{g_{i}}\right)= & a_{i}\left(\sum_{k=1}^{z_{i}} P_{i k} u_{i k}\right)^{2}+b_{i}\left(\sum_{k=1}^{z_{i}} P_{i k} u_{i k}\right)+c_{i} \\
F_{i}\left(P_{g_{i}}\right)= & a_{i}\left(\sum_{k=1}^{z_{i}} P_{i k} u_{i k}\right)^{2}+b_{i}\left(\sum_{k=1}^{z_{i}} P_{i k} u_{i k}\right)+c_{i} \\
& +\left|e_{i} \sin \left(f_{i}\left(\underline{P}_{g_{i}}-\left(\sum_{k=1}^{z_{i}} P_{i k} u_{i k}\right)\right)\right)\right|
\end{aligned}
$$

As it is clear from these objective functions, the decision-making variables are included in the objective function, which makes it adaptable with pre-solving and solving processes of the commercial MINLP solvers.

Therefore, by modifying the mathematical formulations presented in Section 2.1, the SP-MINLP model is obtained. These modifications are (a) replacing $P_{g_{i}}$ in (1) and (2) by (33), and (b) taking into account the (32) and (34).

\subsubsection{Sub-function-based LCOPF}

Another MINLP model can be obtained by assigning a cost function

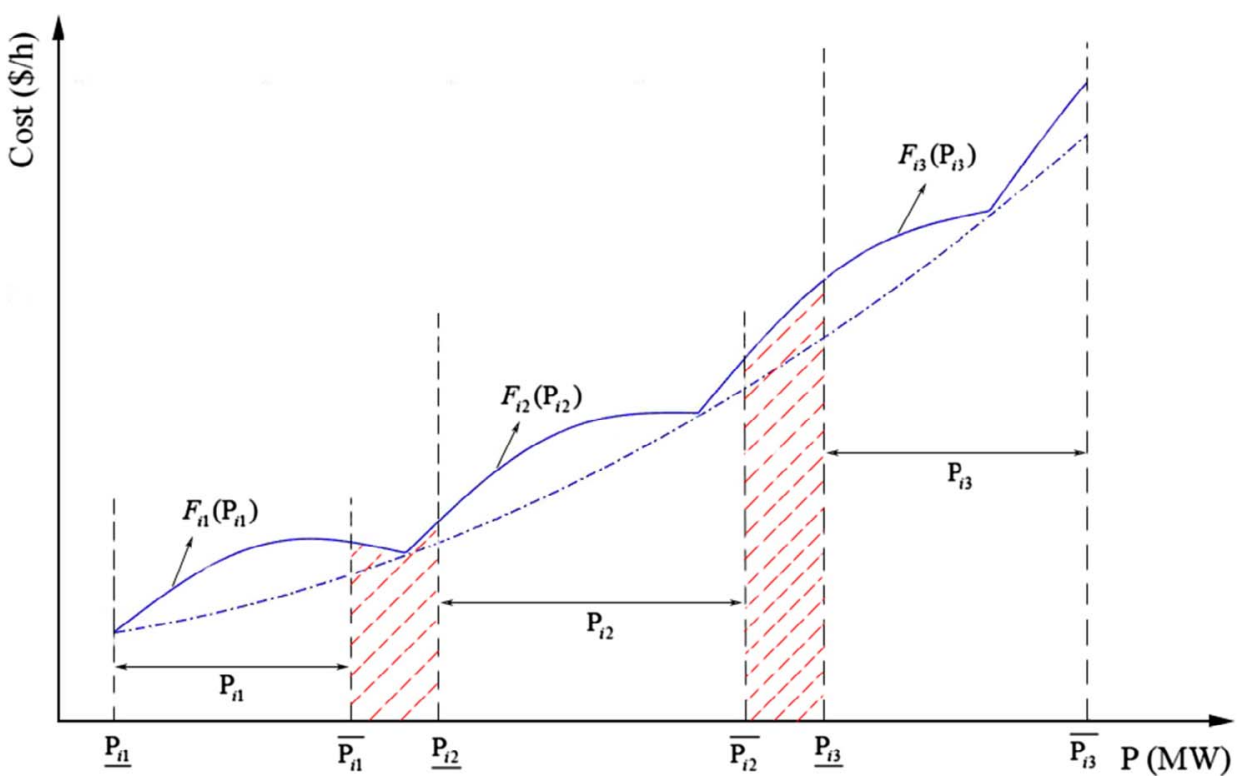

to each disjoint operating zone. Therefore, as it can be seen from Fig. 5, instead of selecting the sub-powers, the sub-cost functions correspond to the operating zones $\left(F_{i 1}\left(P_{i 1}\right), F_{i 2}\left(P_{i 2}\right)\right.$, and $\left.F_{i 3}\left(P_{i 3}\right)\right)$ can be selected. In this regard, the cost function (8) of unit $i$ is defined as a logical subfunction-based objective (37).

$\left\{\begin{array}{l}F_{i 1}\left(P_{i 1}\right)=a_{i} P_{i 1}^{2}+b_{i} P_{i 1}+c_{i}, \text { or } \\ F_{i k}\left(P_{i k}\right)=a_{i} P_{i k}^{2}+b_{i} P_{i k}+c_{i}, \forall 2 \leqslant k \leqslant\left(z_{i}-1\right), \text { or } \\ F_{i z_{i}}\left(P_{i z_{i}}\right)=a_{i} P_{i z_{i}}^{2}+b_{i} P_{i z_{i}}+c_{i}\end{array}\right.$

And the cost function considering the valve-point effects, (9), after the modifications is represented by (38).

$\left\{\begin{array}{l}F_{i 1}\left(P_{i 1}\right)=a_{i} P_{i 1}^{2}+b_{i} P_{i 1}+c_{i}+\left|e_{i} \times \sin \left(\mathrm{f}_{i} \times\left(\underline{P}_{i}-P_{i 1}\right)\right)\right|, \text { or } \\ F_{i k}\left(P_{i k}\right)=a_{i} P_{i k}^{2}+b_{i} P_{i k}+c_{i}+\left|e_{i} \times \sin \left(\mathrm{f}_{i} \times\left(\underline{P}_{i}-P_{i k}\right)\right)\right|, \forall 2 \leqslant k \leqslant\left(z_{i}-1\right), \text { or } \\ F_{i z_{i}}\left(P_{i z_{i}}\right)=a_{i} P_{i z_{i}}^{2}+b_{i} P_{i z_{i}}+c_{i}+\left|e_{i} \times \sin \left(\mathrm{f}_{i} \times\left(\underline{P}_{i}-P_{i z_{i}}\right)\right)\right|\end{array}\right.$

By reformulating the aforementioned logical sub-functions, (37) and (38), to an equivalent MINLP model, the sub-function-based MINLP (SFMINLP) for solving LCOPF problems is obtained as follows.

Minimize $\sum_{i \in \Omega_{g}} \sum_{k=1}^{z_{i}}\left(F_{i k}\left(P_{i k}\right)\right) u_{i k}$

In order to guarantee that only one of the decision-making variables $u_{i k}$ can be set to $1,(40)$ is taken into account, and consequently, only one of the sub-functions can be selected.

$\sum_{k=1}^{z_{i}} u_{i k}=1, \quad \forall u_{i k} \in\{0,1\}$

However, to obtain the mixed-integer programming model, some more definition such as (41) is necessary while the $P_{g_{i}}$ in (2) must be replaced by (42).

$\underline{P}_{i \mathrm{k}} \leqslant P_{i \mathrm{k}} \leqslant \bar{P}_{i \mathrm{k}} ; \forall i \in \Omega_{\mathrm{g}}, k \in\left\{1, \ldots, z_{i}\right\}$

$P_{g_{i}}=\sum_{k=1}^{z_{i}} P_{i k} u_{i k}$

Therefore, by making some modifications in the mathematical formulations presented in Section 2.1, SF-MINLP model is obtained. These modifications are (a) replacing the objective function (1) with a subfunction-based objective (37) or (38), (b) putting (42) in (2), and (c) considering (40) and (41).

Fig. 5. The input-output curve of unit $i$ with considering valve-point effect and POZs. 


\section{Case studies and results}

In order to verify and validate the proposed models, the results of testing on a commonly used IEEE 30-bus system under different topologies and conditions are compared with the existing works in the literature. However, to show the effectiveness, usefulness, and the computational tractability and efficiency of the proposed models in facing with logically-constrained large-scale power systems and also to compare their potential with each other, two systems such as IEEE 118bus and Polish 2383-bus are studied. In this paper, to implement the proposed models, a modeling language for mathematical programming (AMPL) is used and to solve the proposed mixed integer nonlinear programming models via a 3.3-GHz computer with $3 \mathrm{~GB}$ of RAM. After testing the models by different MINLP solvers such as Localsolver, Counne, Bonmin, and Knitro, we concluded that the nonlinear commercial solvers Knitro is the most reliable and efficient solver for our proposed models [37]. Since the model has been created to be adaptable to the pre-solving techniques of commercial solvers, the pre-solve option of Knitro that is on by default is remained on, otherwise, it can be disregarded by setting the user option "presolve $=0$ ". It is worth mentioning that, Knitro uses a MIP heuristic search approach to find the initial integer feasible points. Once the initial integer feasible points have been defined, the branch-and-bound procedure is started [37]. Moreover, in order to compare the CPU times of different approaches, in this paper the adjusted CPU time (43) is used [38].

$A C T=\frac{\text { given } \mathrm{CPU} \text { speed }(\mathrm{GHz})}{3.3 \mathrm{GHz}} \times \frac{\text { given CPU time }(\mathrm{s})}{\mathrm{CPU} \text { time from MINLPs }(\mathrm{s})}$

\subsection{IEEE 30-bus test system}

The IEEE 30-bus system, among the other systems in the literature, is a commonly used system that has been tested under different conditions and topologies. In some works, the LCOPF with shunt VAR compensator and LTCT have been considered, while in some other works the LCOPF with TCSC and TCPS have been studied. In this paper, to verify and show the effectiveness of the proposed MINLP frameworks, both the aforementioned configurations via the corresponding formulations (second and third models) in Section 2.1, are studied. This system consists of 30 buses ( 9 buses with shunt VAR compensator), 41 branches (4 branches with transformer), and 6 generating units [39]. For this system, four different cases are considered. The detail data of cases 1-3 and POZs are obtained from [10], while the detail data of case 4 is obtained from [36]. The minimum and maximum bounds of voltages at load buses are 0.95 p.u. and 1.05 p.u. while for the generator buses are 0.95 p.u. and 1.1 p.u., respectively. The minimum and maximum bounds for the transformer tap setting are 0.9 p.u. and 1.1 p.u with the step size of 0.0125 p.u.; the minimum and maximum bounds of shunt VAr compensators are 0.0 p.u. and 0.05 p.u with the step size of 0.01 p.u.; the minimum and maximum limits of reactance of TCSCs are 0 p.u. and 0.034 p.u; and the minimum and maximum limits of phase shift angles of TCPS are -5 and 5 degrees, respectively.

\subsubsection{Case 1: F2 considering prohibited operating zone (POZ)}

This case is used to validate the proposed SP-MINLP and SF-MINLP models in facing with POZs comparing with several existing approaches in the literature. Table 1 presents the optimal results of proposed models and the other existing approaches in the literature such as Genetic Algorithm (GA) [40], Particle Swarm Optimization (PSO) [40], Simulated Annealing (SA) [40], Shuffle Frog Leaping Algorithm (SLFA) [40], Hybrid SFLA and SA (HSLFA-SA) [40], Enhanced GA (EGA) $[41,10]$, and three Efficiency Improvement Metaheuristic Algorithms (EIMA1, EIMA2, and CEIMA) [10] where in EIMA1 a hybrid EGA Combined with a Successive Approximation using Linear Programming (SALP) or Quadratic Programming (QP) is used, while the EIMA2 is performed by determining the lower and upper bounds, and CEIMA is a combination of EIMA1 and EIMA2 approaches. It is worth mentioning that the result of SF-MINLP model is used as the basis of per unit calculations of adjusted CPU time (ACT).

As it can be seen from Table 1, the best result in the literature was obtained by HSLFA-SA with a total cost of $\$ 805.8 / \mathrm{h}$ within $22.19 \mathrm{~s}$ (2.38 p.u.) and considering the CPU times shows that the three approaches presented in [10] such as EIMA1, EIMA2, and CEIMA, compared to other approaches in the literature as well as the proposed SPMINLP and SF-MINLP models, have higher computational efficiencies where the CEIMA, with the best CPU time among all, requires only 0.49 p.u to find an optimal solution. However, the price of obtaining this computational efficiency was reducing the quality of optimal solution where the results of these approaches are not even as good as the HSLFA-SA. On the other hand, the optimal results show that the proposed SP-MINLP and SF-MINLP models have obtained much better optimal solution than other approaches with $\$ 800.7 / \mathrm{h}$, which is $\$ 5.1 / \mathrm{h}$ less than the best result in the literature. Results also show that the proposed SF-MINLP model, comparing with the SP-MINLP that obtains the same result as the SF-MINLP model, is about 5.65 times faster, and comparing with the CEIMA, which is the fastest among all, obtains a much higher quality solution, with $\$ 6.0 / \mathrm{h}$ fewer cost. Comparing the proposed models show that the number of nodes and subproblems to be solved to obtain the optimal solution of the problem via SF-MINLP model are 235 and 238, while via the SP-MINLP model these numbers are 882 and 1062, respectively, which are much higher than those numbers resulted via the SF-MINLP model.

\subsubsection{Case 2: F2 considering POZ and valve-point effect (VPE)}

Considering POZs and VPE simultaneously brings more difficulties for the nonlinear solver, however, we use this model to reveal the potential of the proposed models in facilitating the solver's processes in finding an optimal solution.

Table 2 presents the comparison among the optimal solutions of the proposed models and other existing approaches in the literature. The optimal solution of the proposed models is $\$ 828.1 / \mathrm{h}$, which is much better than the best optimal solution in the literature, which belongs to

Table 1

Comparison of the Optimal Results of the Proposed Models with Other Approaches for IEEE 30-bus System considering POZ.

\begin{tabular}{|c|c|c|c|c|c|c|c|c|c|}
\hline Methods & $\mathrm{P}_{\mathrm{g} 1}(\mathrm{MW})$ & $\mathrm{P}_{\mathrm{g} 2}(\mathrm{MW})$ & $\mathrm{P}_{\mathrm{g} 5}(\mathrm{MW})$ & $\mathrm{P}_{\mathrm{g} 8}(\mathrm{MW})$ & $\mathrm{P}_{\mathrm{g} 11}(\mathrm{MW})$ & $\mathrm{P}_{\mathrm{g} 13}(\mathrm{MW})$ & Cost $(\$ / h)$ & Time (s) & ACT \\
\hline GA $[40]$ & 175.1155 & 44.5947 & 23.392 & 23.6505 & 11.5278 & 15.9364 & 809.2 & NA & NA \\
\hline PSO [40] & 174.2634 & 57.0264 & 23.5392 & 14.1558 & 11.003 & 14.5145 & 806.4 & 24.94 & 2.68 \\
\hline SA [40] & 181.1696 & 57.9178 & 17.1063 & 16.5193 & 10 & 12 & 808.7 & 123.86 & 13.29 \\
\hline SLFA $[40]$ & 182.8236 & 45 & 21.0399 & 20.4853 & 12.0338 & 13.3021 & 806.2 & 24.74 & 2.66 \\
\hline HSLFA-SA [40] & 181.453 & 45 & 21.5269 & 22.0786 & 11.9854 & 12 & 805.8 & 22.19 & 2.38 \\
\hline EGA $[10]$ & 177.6082 & 44.4762 & 22.3846 & 18.84 & 15.3382 & 13.7368 & 806.5 & 33.0397 & 4.32 \\
\hline EIMA1 [10] & 182.9833 & 42.315 & 21.6154 & 20.5372 & 11.0452 & 14.1265 & 806.9 & 6.4699 & 0.85 \\
\hline EIMA2 [10] & 178.311 & 44.5311 & 20.6239 & 24.1148 & 12.5788 & 12.2325 & 806.7 & 7.5270 & 0.98 \\
\hline CEIMA [10] & 177.6929 & 44.8573 & 19.0112 & 21.5042 & 16.4863 & 14.4460 & 806.7 & 3.7395 & 0.49 \\
\hline SP-MINLP & 179.309 & 45 & 21.5328 & 22.3159 & 12.26 & 12.0001 & 800.7 & 42.451 & 5.65 \\
\hline SF-MINLP & 179.311 & 45 & 21.5323 & 22.3132 & 12.2607 & 12.0001 & 800.7 & 7.512 & 1.00 \\
\hline
\end{tabular}


Table 2

Comparison of the optimal results the proposed models with other approaches for IEEE 30-bus System considering POZ and VPE.

\begin{tabular}{|c|c|c|c|c|c|c|c|c|c|}
\hline Methods & $\mathrm{P}_{\mathrm{g} 1}(\mathrm{MW})$ & $\mathrm{P}_{\mathrm{g} 2}(\mathrm{MW})$ & $\mathrm{P}_{\mathrm{g} 5}(\mathrm{MW})$ & $\mathrm{P}_{\mathrm{g} 8}(\mathrm{MW})$ & $\mathrm{P}_{\mathrm{g} 11}(\mathrm{MW})$ & $\mathrm{P}_{\mathrm{g} 13}(\mathrm{MW})$ & Cost $(\$ / h)$ & Time (s) & ACT \\
\hline GA [40] & 226.1783 & 24.3344 & 16.9393 & 10.00 & 10.1525 & 12.00 & 838.2 & $\mathrm{Na}$ & $\mathrm{Na}$ \\
\hline PSO [40] & 221.1471 & 30.8367 & 15.00 & 10.00 & 10.00 & 12.00 & 835.5 & 31.62 & 9.26 \\
\hline SA [40] & 217.6117 & 32.7708 & 16.5177 & 10.00 & 10.00 & 12.00 & 836.5 & 152.32 & 44.60 \\
\hline SLFA $[40]$ & 219.4201 & 31.1173 & 15.8535 & 10.0415 & 10.00 & 12.00 & 834.8 & 30.72 & 8.99 \\
\hline HSLFA-SA [40] & 219.816 & 29.7707 & 16.667 & 10.00 & 10.00 & 12.00 & 834.6 & 27.57 & 8.07 \\
\hline EGA $[10]$ & 219.3148 & 24.7033 & 17.7778 & 13.3211 & 10.337 & 12.00 & 835.2 & 36.8067 & 13.13 \\
\hline EIMA1 [10] & 199.3421 & 44.00 & 15.5556 & 10.1465 & 12.7057 & 12.9709 & 835.8 & 7.1784 & 2.56 \\
\hline EIMA2 [10] & 219.1556 & 24.0293 & 15.1111 & 10.1954 & 12.7106 & 15.535 & 835.2 & 8.3179 & 2.97 \\
\hline CEIMA [10] & 206.6835 & 32.5275 & 15.5214 & 16.3309 & 11.6606 & 12.4991 & 835.2 & 4.1280 & 1.47 \\
\hline SP-MINLP & 212.499 & 30.2251 & 17.9767 & 11.5671 & 10.6367 & 12.0011 & 828.1 & 7.354 & 2.67 \\
\hline SF-MINLP & 212.421 & 30.2414 & 18.0011 & 11.4883 & 10.7416 & 12.0011 & 828.1 & 2.753 & 1.00 \\
\hline
\end{tabular}

HSLFA-SA with $\$ 834.6 / \mathrm{h}$. This means that by using the proposed models, the generation side will decrease the costs by about $\$ 6.5 / \mathrm{h}$, which is an acceptable decrease for such small scale system via the optimization tool. Moreover, results show that the SF-MINLP model not only capable of obtaining a better optimal solution but also shows higher computational efficiency than the fastest approach in the literature, CEIMA with 1.47 p.u ACT, and the other proposed model, SPMINLP with 2.67 p.u. ACT. Therefore, the SF-MINLP model by performing an acceptable trade-off between model accuracy and complexity facilitates finding the optimal solution for the nonlinear solver. It is worth mentioning that the numbers of nodes and solved subproblems for SF-MINLP model are 1737 and 1774, while for the SPMINLP model are 1864 and 2249, respectively. This shows that for this case, SP-MINLP model faces with more difficulties to obtain the optimal solution by solving 475 subproblems more than the SF-MINLP model.

\subsubsection{Case 3: F2 with loss minimization objective}

Although in this case the POZ is not considered, based on the existing integer decision-making variables related to LTCTs and shunt VAr compensators, (18) and (19), the problem is still a highly mixed-integer nonlinear programming problem. In this case, because there is no disjoint operating zone, the SP-MINLP and SF-MINLP models are the same, however, we use this case to show the flexibility and potential of the proposed models in facing with different types of mixed-integer based problems under different system configurations.

Table 3 presents the optimal solution of different approaches by providing the active power output, voltages of generator buses, transformers' tap settings, shunt VAr compensator settings, total cost, system loss, and the CPU time. As it is clear from this table, the proposed MINLP model obtains a better optimal solution, with 3.0911 MW transmission loss, than the best optimal solution reported in the literature which belongs to DE with $3.1315 \mathrm{MW}$ transmission loss. Therefore, the transmission loss obtained by the DE approach, compared with the MINLP model, is about 1.3\% higher yet it requires about 184.96 times more CPU time to converge [10]. Comparing the convergence velocity of the MINLP model shows its superiority over the other approaches in the literature where it is 5.10 times faster than the fastest approach, the CEIMA with $3.3109 \mathrm{~s}$ [10]. Moreover, the detailed results verify that the MINLP model by providing a different pattern for shunt VAr compensator and LTCTs performs a more appropriate dispatch, and consequently the loss is decreased. This shows that how an appropriate tradeoff between the model accuracy and complexity helps

Table 3

Comparison of the optimal results of the minlp model with other approaches for IEEE 30-bus system, loss minimization objective.

\begin{tabular}{|c|c|c|c|c|c|c|c|c|c|}
\hline Output & PSO [42] & EEA [42] & GA [42] & EIMA2 [10] & CEIMA [10] & EGA [42] & EGA-DQLF [43] & DE [10] & MINLP \\
\hline $\mathrm{P}_{\mathrm{g} 1}(\mathrm{MW})$ & 56.6613 & 59.3216 & 56.1602 & 65.7984 & 63.3470 & 51.674 & 51.6008 & 51.9581 & 51.4912 \\
\hline $\mathrm{P}_{\mathrm{g} 2}(\mathrm{MW})$ & 78.9597 & 74.8132 & 77.82 & 71.003 & 69.5824 & 79.97 & 80 & 79.9121 & 80.0 \\
\hline $\mathrm{P}_{\mathrm{g} 5}(\mathrm{MW})$ & 49.1795 & 49.8547 & 49.94 & 49.8547 & 49.6667 & 50 & 50 & 49.9743 & 50.0 \\
\hline $\mathrm{P}_{\mathrm{g} 8}(\mathrm{MW})$ & 35 & 34.9084 & 34.75 & 33.0464 & 34.8169 & 35 & 35 & 34.8657 & 35.0 \\
\hline $\mathrm{P}_{\mathrm{g} 11}(\mathrm{MW})$ & 29.8242 & 28.1099 & 29.897 & 27.3773 & 29.9170 & 30 & 30 & 29.8339 & 30.0 \\
\hline $\mathrm{P}_{\mathrm{g} 13}(\mathrm{MW})$ & 37.094 & 39.7538 & 38.11 & 39.5897 & 39.3299 & 40 & 40 & 39.9863 & 40.0 \\
\hline $\mathrm{V}_{1}$ (p.u.) & 1.0694 & 1.0547 & 1.058 & 1.0912 & 1.0918 & 1.0518 & 1.0435 & 1.06 & 1.06317 \\
\hline $\mathrm{V}_{2}$ (p.u.) & 1.0729 & 1.0418 & 1.051 & 1.0812 & 1.0882 & 1.0488 & 1.04353 & 1.0558 & 1.05675 \\
\hline $\mathrm{V}_{5}$ (p.u.) & 1.05 & 1.0247 & 1.034 & 1.0735 & 1.0629 & 1.027 & 1.02470 & 1.0341 & 1.03730 \\
\hline $\mathrm{V}_{8}$ (p.u.) & 1.0476 & 1.0335 & 1.042 & 1.0759 & 1.0835 & 1.0306 & 1.03470 & 1.04 & 1.04362 \\
\hline $\mathrm{V}_{11}$ (p.u.) & 1.0176 & 1.0229 & 1.089 & 1.0559 & 1.02 & 1.0612 & 1.07 & 1.0588 & 1.07978 \\
\hline $\mathrm{V}_{13}$ (p.u.) & 1.0576 & 1.0776 & 1.042 & 1.0924 & 1.0988 & 1.0382 & 1.043 & 1.0941 & 1.05098 \\
\hline $\mathrm{T}_{6-9}$ (p.u.) & 0.95 & 1.0125 & 1.0625 & 1.025 & 0.9875 & 1.075 & 1.0375 & 0.95 & 0.95 \\
\hline $\mathrm{T}_{6-10}$ (p.u.) & 1.0125 & 0.9125 & 1.0125 & 0.9375 & 0.9250 & 0.95 & 0.925 & 0.95 & 1.0875 \\
\hline $\mathrm{T}_{4-12}$ (p.u.) & 0.9875 & 1.0125 & 1.025 & 1.0125 & 1.0250 & 0.9875 & 0.975 & 0.925 & 1.0125 \\
\hline $\mathrm{T}_{28-27}$ (p.u.) & 1.0375 & 1.0125 & 1.0125 & 0.975 & 1.00 & 1.0125 & 0.975 & 1.0625 & 1.025 \\
\hline $\mathrm{Q}_{\mathrm{C} 10}$ (p.u.) & 0.05 & 0.04 & 0.04 & 0.05 & 0.02 & 0.04 & 0.05 & 0.05 & 0.01 \\
\hline $\mathrm{Q}_{\mathrm{c} 12}$ (p.u.) & 0.05 & 0.02 & 0.03 & 0.04 & 0.04 & 0.04 & 0.03 & 0.00 & 0.03 \\
\hline $\mathrm{Q}_{\mathrm{C} 15}$ (p.u.) & 0.05 & 0.05 & 0.05 & 0.05 & 0.00 & 0.02 & 0.0 & 0.03 & 0.05 \\
\hline $\mathrm{Q}_{\mathrm{C} 17}$ (p.u.) & 0.03 & 0.01 & 0.02 & 0.02 & 0.05 & 0.05 & 0.01 & 0.04 & 0.05 \\
\hline $\mathrm{Q}_{\mathrm{C} 20}$ (p.u.) & 0.04 & 0.05 & 0.04 & 0.01 & 0.04 & 0.03 & 0.04 & 0.00 & 0.04 \\
\hline $\mathrm{Q}_{\mathrm{C} 21}$ (p.u.) & 0.05 & 0.0 & 0.02 & 0.01 & 0.02 & 0.01 & 0.02 & 0.04 & 0.05 \\
\hline $\mathrm{Q}_{\mathrm{C} 23}$ (p.u.) & 0.02 & 0.02 & 0.05 & 0.05 & 0.02 & 0.0 & 0.05 & 0.01 & 0.03 \\
\hline $\mathrm{Q}_{\mathrm{C} 24}$ (p.u.) & 0.0 & 0.05 & 0.0 & 0.02 & 0.05 & 0.03 & 0.05 & 0.0 & 0.05 \\
\hline $\mathrm{Q}_{\mathrm{C} 29}$ (p.u.) & 0.01 & 0.02 & 0.0 & 0.04 & 0.01 & 0.04 & 0.05 & 0.0 & 0.02 \\
\hline Cost $(\$ / h)$ & 954.3483 & 952.3785 & 957.84 & 940.9685 & 944.7613 & 967.93 & 967.86 & 966.7930 & 967.592 \\
\hline Losses (MW) & 3.318 & 3.2823 & 3.2772 & 3.2695 & 3.2601 & 3.244 & 3.2008 & 3.1315 & 3.0912 \\
\hline Time (s) & NA & 5.7167 & NA & 6.9117 & 3.3109 & 29.7118 & NA & 120.0 & 0.637 \\
\hline ACT & NA & 8.81 & NA & 10.65 & 5.10 & 45.79 & NA & 184.96 & 1.00 \\
\hline
\end{tabular}


in proper settings and accordingly finding a better optimal solution. It should be noted that to obtain the optimal solution the numbers of nodes and subproblems to be solved are 56 and 57, respectively.

\subsubsection{Case 4: F3 considering POZ and VPE}

Unlike the other three cases, this case considers the TCSC and the TCPS devices. Consequently, as it can be seen from formulation F3 in Section 2.1, in regards with non-convexity and nonlinearity, the problem is more complicated than the problem of F1 and F2. It is relevant to mention that this case is used to validate and examine the performance (obtaining an optimal solution and computational efficiency) of the proposed SP-MINLP and SF-MINLP models in solving highly nonlinear and nonconvex mixed-integer problems.

Table 4 presents the results of the proposed models for LCOPF problems considering FACTS devices. Since the qualities of optimal results are almost the same, to show the efficiency of the proposed models, the decision making variables, CPU time, and solving process are considered in details. Considering the output of generating units obtained by SF-MINLP model, which has a negligible higher quality result, shows that several units such as $1,8,11$, and 13 are adjusted to their lower or upper limits of an operating zone, however, the SPMINLP model fails of such precise adjustments. This failure is a result of difficulties in finding a precise adjustment of TCSC and TCPS control variables in which negatively, although negligible, affects in generation costs. Such difficulties become even clearer by considering the CPU times, the number of nodes to be probed, and the number of subproblems to be solved. The SF-MINLP model finds the optimal solution at the first node and by solving only three subproblems. This shows the potential of SF-MINLP model to facilitate the pre-solving and probing techniques of commercial solver that results in converging to an optimal solution within $0.187 \mathrm{~s}$. On the other hand, the SP-MINLP model probes 235 nodes and solves 238 subproblems to converge to an optimal solution within $9.547 \mathrm{~s}$, which is about 51 times slower than the SF-MINLP model. This shows the importance of using an appropriate model for MINLP problems in finding a more precise optimal solution with a higher computational efficiency. The results of this case demonstrate the superiority of SF-MINLP model in solving such highly non-convex and nonlinear LCOPF problems considering multiple control devices.

\subsection{IEEE 118-bus system}

This system consists of 118 buses, 186 branches, and 54 generators where 20 out of 54 generating units have a total 42 prohibited operating zones. For this system, the lower and upper limits of LTCTs are, respectively, 0.9 p.u. and 1.1 p.u. and shunt VAr compensators can vary between 0 and 0.05 p.u. It is worth mentioning that, in this case, for the sake of simplicity the tap and VAr control variables are considered to be continuous. The data of this system is obtained from [44], and the additional data of POZs, buses with shunt VAr compensator and branches with LTCT are provided in the appendix, Tables A1 and A3, respectively.

The optimal solutions of this system that contains 62 disjoint operating zones by SP-MINLP and SF-MINLP models are almost the same, about $\$ 129,619.67 / \mathrm{h}$. Therefore, to reveal the efficiency and potential of the proposed models, the details of solving process is taken into consideration. After the pre-solving process of Knitro solver, the number of nonzero elements in Hessians for SP-MINLP and SF-MINLP models are, respectively, 1584 and 1320. Although these numbers show that the SF-MINLP method may need fewer calculations and acts better in the pre-solving process, the model simplicity will be revealed after considering the solving process. Results show that, for branch and bound method of Knitro, the number of nodes to be probed and subproblems to be solved for SP-MINLP are 121 and 122, respectively, while via SF-MINLP model only five nodes and five subproblems are processed. Moreover, the SF-MINLP model needs only $4.3 \mathrm{~s}$ to find the optimal solution, while the CPU time of SP-MINLP model is $24.87 \mathrm{~s}$, which is about 4.87 times slower than SF-MINLP model. This demonstrates the superiority of the SF-MINLP model for solving systems with many disjoint operating zones.

\subsection{Polish 2383-bus system}

The Polish system, which is a very large-scale power system, contains 2383 buses, 2896 branches, 327 generators with 24 POZs, and 1826 load buses with 24558.4 MW and 8143.9 MVAr active and reactive demands, respectively. By taking into account the LTCTs and VAr compensators, this large-scale system becomes a very complicated optimization problem. In this system, the lower and upper limits of LTCTs are, respectively, 0.9 p.u. and 1.1 p.u. and shunt VAr compensators can vary between 0 and 0.05 p.u. while the tap and VAr control variables are continuous. The data of this system is obtained from [44] while the additional data of POZs are provided in the appendix, Tables A2 and A3. In order to reveal the strength and drawbacks of our proposed models, this system is studied under four different configurations, shown in Table 5. The POZ is considered for all cases while the combination of shunt VAR compensators, LTCT, and line limit makes the other system configurations to be considered.

Results from Table 5 show that, for the first case, the total generation cost obtained by SF-MINLP is $\$ 1,860,059.9 / \mathrm{h}$, which is $\$ 103.8 / \mathrm{h}$ less than the result obtained by SP-MINLP model, yet the SP-MINLP model requires $117.07 \mathrm{~s}$ more $\mathrm{CPU}$ time to converge. Considering the optimal solutions of cases 2,3 , and 4 demonstrate that both of the proposed models have found the same generation costs. It is worth mentioning that, for the third case in which the line limits are considered, although the SP-MINLP model finds the same solution as the SF-MINLP model, its integrality gap is -695 , and for a solution to such largely negative integrality gap the Knitro solver is unable to verify the optimality even if it claims [37]. Moreover, for this case, the SP-MINLP problem even with probing fewer nodes and solving fewer subproblems shows less computational efficiency, with 1201.48 s, where comparing to the SF-MINLP model it needs $566.45 \mathrm{~s}$ more CPU time to converge. Considering case 4 in which among all cases more decision variables should be adjusted shows a higher computational efficiency of SFMINLP by requiring only $150.94 \mathrm{~s}$, while the SP-MINLP model with $716.08 \mathrm{~s}$ CPU time is 4.74 times slower than it. This shows the superiority of the SF-MINLP model in bringing more facilities for the commercial nonlinear solver in finding an optimal solution for highly complex systems.

Table 4

Comparison the units' outputs and FACTS adjustments for SP-MINLP and SF-MINLP models, IEEE 30-bus system.

\begin{tabular}{|c|c|c|}
\hline Optimal Output & SP-MINLP & SF-MINLP \\
\hline $\mathrm{P}_{\mathrm{g} 1}(\mathrm{MW})$ & 199.999999 & 200.00 \\
\hline $\mathrm{P}_{\mathrm{g} 2}(\mathrm{MW})$ & 43.376381 & 43.376388 \\
\hline $\mathrm{P}_{\mathrm{g} 5}(\mathrm{MW})$ & 18.627491 & 18.627488 \\
\hline $\mathrm{P}_{\mathrm{g} 8}(\mathrm{MW})$ & 10.000003 & 10.00 \\
\hline $\mathrm{P}_{\mathrm{g} 11}(\mathrm{MW})$ & 10.000002 & 10.00 \\
\hline $\mathrm{P}_{\mathrm{g} 13}(\mathrm{MW})$ & 12.000001 & 12.00 \\
\hline$x_{3-4}^{c}$ (p.u.) & 0.0325116 & 0.0325137 \\
\hline$x_{19-20}^{c}$ (p.u.) & 0.0339927 & 0.0339999 \\
\hline$\varphi_{5-7}$ (deg.) & 0.0364783 & 0.0364786 \\
\hline$\varphi_{10-22}$ (deg.) & -0.00068501 & -0.00068499 \\
\hline Cost $(\$ / h)$ & 831.069700 & 831.069696 \\
\hline Loss (MW) & 10.603877 & 10.603876 \\
\hline Time (s) & 9.547 & 0.187 \\
\hline \# of nodes & 235 & 1 \\
\hline \# of subproblems & 238 & 3 \\
\hline
\end{tabular}


Table 5

Results of different cases for polish 2383-bus system.

\begin{tabular}{|c|c|c|c|c|c|c|c|c|}
\hline Case & Method & Cost $(\$ / h)$ & Time (s) & \# of nodes & $\#$ of sub- problems & POZ & LTCT \& VAR & Line Limit \\
\hline \multirow[t]{2}{*}{ Case 1} & SP-MINLP & 1860163.7 & 358.60 & 7 & 9 & $\checkmark$ & & - \\
\hline & SF-MINLP & 1860059.9 & 241.53 & 6 & 7 & & & \\
\hline \multirow[t]{2}{*}{ Case 2} & SP-MINLP & 1858989.7 & 387.6 & 7 & 9 & $\checkmark$ & $\checkmark$ & - \\
\hline & SF-MINLP & 1858989.7 & 368.8 & 7 & 9 & & & \\
\hline \multirow[t]{2}{*}{ Case 3} & SP-MINLP & $1906024.4^{\mathrm{a}}$ & 1201.48 & 10 & 11 & $\checkmark$ & - & $\checkmark$ \\
\hline & SF-MINLP & 1906024.4 & 635.03 & 11 & 12 & & & \\
\hline \multirow[t]{2}{*}{ Case 4} & SP-MINLP & 1893506.0 & 716.08 & 12 & 14 & $\checkmark$ & $\checkmark$ & $\checkmark$ \\
\hline & SF-MINLP & 1893506.0 & 150.94 & 12 & 14 & & & \\
\hline
\end{tabular}

${ }^{\text {a }}$ The integrality gap is largely negative.

\section{Conclusion}

From the practical standpoint, the solver-based models are of much interest. On the other hand, the existing commercial nonlinear solvers are not capable of solving logic-based problems. In this paper, by considering the pre-solving and solving processes of the commercial nonlinear solver, the logical constraints have been recast into the terms of objective function. These recasts may facilitate the pre-solving and probing techniques of the commercial solver and consequently, results in computational and solution efficiency. By recasting the logical constraints to sub-powers corresponding to the disjoint operating zones, the SP-MINLP model obtains, while by recasting them into sub-functions, the SF-MINLP model results. The proposed models, as the first solver-friendly models for nonconvex logically-constrained OPF problems, have addressed the existing shortcoming in the literature for solving such problems by commercial solvers. Results show that the proposed models are much better than, faster with a higher potential for finding a high-quality optimal solution, the existing approaches in the literature. It is relevant to highlight the effectiveness and usefulness of the proposed models in finding the optimal solution for very large- scale practical Polish power system under different conditions and system configurations. Comparing to the existing approaches in the literature, which all of them are heuristic-based, to solve logically constrained ACOPF problems, our well-defined solver-friendly MINLP models, by facilitating the pre-solving and solving processes, show higher performance and efficiency in finding an optimal solution. However, the SF-MINLP model by bringing more facilities for nonlinear solver can be the best option for companies for solving such highly nonlinear non-convex mixed-integer logically constrained OPF problems as the SP-MINLP model fails in finding a high-quality solution where the SF-MINLP model finds. All in all, it can be deduced that although a nonlinear solver may not guarantee to find the globally optimal solution, a well-defined model via an efficient recast method results in finding a much higher quality solution than the heuristic-based approaches.

\section{Acknowledgements}

This work was supported by FAPESP (No. 2014/22828-3, and No. 2016/14319-7), CAPES, and CNPq (No. 305371/2012-6).

\section{Appendix A}

This appendix contains the necessary data for POZs, buses with shunt VAR compensator, and branches with LTCT for IEEE 118-bus and Polish 2383-bus systems, Tables A1-A3.

Table A1

POZs for IEEE 118-bus system.

\begin{tabular}{ll}
\hline Buses with POZ & POZs \\
\hline $1,4,6,15,34,70$ & $(2030)(6085)$ \\
10 & $(1545)(165200)(395410)$ \\
25 & $(4065)(190200)$ \\
26 & $(7595)(260280)$ \\
$40,42,85,104,116,99$ & $(2030)(4555)$ \\
49 & $(4560)(185200)$ \\
59 & $(95105)(140155)$ \\
61 & $(145155)(210230)$ \\
65 & $(180200)(350360)$ \\
89 & $(120145)(410460)(500525)$ \\
\hline
\end{tabular}

Table A2

POZs for polish 2383-bus system.

\begin{tabular}{llll}
\hline Bus with POZ & POZs & Bus with POZ \\
\hline 18 & $(18501950)(20202200)$ & 139 & $(410490)(560580)$ \\
95 & $(5565)(7590)$ & 476 & $(470510)(580598)$ \\
123 & $(2545)(7590)$ & 755 & $(224227)(229231)$ \\
125 & $(160175)(180190)$ & 1182 & $(8792)(130160)$ \\
131 & $(865885)(890930)$ & 1416 & $(4550)(95102)$ \\
132 & $(7285)(145190)$ & $(320335)(355360)$ \\
\hline
\end{tabular}


Table A3

Branches with LTCT and buses with Shunt VAr compensator for IEEE 118- and polish 2383-bus systems.

\begin{tabular}{|c|c|c|}
\hline System & Branches with LTCT & Buses with VAR \\
\hline 118-bus & (8-5), (26-25), (30-17), (38-37), (63-59), (64-61), (65-66), (68-69), (81-80) & $5,17,33,37,44,45,47,48,75,79,82,83,106,108,109$ \\
\hline 2383-bus & $\begin{array}{l}(355-1),(220-3),(309-5),(321-7),(347-10),(366-13),(17-18),(502-21), \\
(568-26),(41-43),(778-55),(821-59),(834-60),(1184-86),(1536-103), \\
(1760-123),(1764-123),(1761-124),(1763-124),(2186-164),(1764-1760) \\
(1763-1761)\end{array}$ & $\begin{array}{l}36,75,76,92,105,106,115,116,137,138,145,146,165,180,185,189, \\
443,513,1690,1699,1726,1756,1766,1793,1794,1796,1878,1879, \\
1905,1935,2146,2189\end{array}$ \\
\hline
\end{tabular}

\section{References}

[1] Bakirtzis AG, Biskas PN, Zoumas CE, Petridis V. Optimal power flow by enhanced genetic algorithm. IEEE Trans Power Syst 2002;17:229-36. http://dx.doi.org/10. 1109/TPWRS.2002.1007886.

[2] Adhvaryyu PK, Chattopadhyay PK, Bhattacharya A. Dynamic optimal power flow of combined heat and power system with Valve-point effect using Krill Herd algorithm. Energy 2017;127:756-67. http://dx.doi.org/10.1016/j.energy.2017.03.046.

[3] Niknam T, Azizipanah-Abarghooee R, Narimani MR. Reserve constrained dynamic optimal power flow subject to valve-point effects, prohibited zones and multi-fuel constraints. Energy 2012;47:451-64. http://dx.doi.org/10.1016/j.energy.2012.07. 053.

[4] Ziaee O, Choobineh F. Optimal location-allocation of TCSCs and transmission switch placement under high penetration of wind power. IEEE Trans Power Syst 2017;32:3006-14. http://dx.doi.org/10.1109/TPWRS.2016.2628053.

[5] Ziaee O, Choobineh FF. Optimal location-allocation of TCSC devices on a transmission network. IEEE Trans Power Syst 2017;32:94-102. http://dx.doi.org/10 1109/TPWRS.2016.2556424.

[6] Basu M. Optimal power flow with FACTS devices using differential evolution. Int J Electr Power Energy Syst 2008;30:150-6. http://dx.doi.org/10.1016/j.ijepes.2007. 06.011.

[7] Padiyar KR. FACTS controllers in power transmission and distribution. New Age International Publishers; 2007.

[8] Huang CM, Huang YC. Hybrid optimisation method for optimal power flow using flexible AC transmission system devices. IET Gener Transm Distrib 2014;8:2036-45. http://dx.doi.org/10.1049/iet-gtd.2014.0096.

[9] Tan Y, Li C, Cao Y, Lee KY, Li L, Tang S, et al. Improved group search optimization method for optimal power flow problem considering valve-point loading effects. Neurocomputing 2015;148:229-39. http://dx.doi.org/10.1016/j.neucom.2013.09. 065 .

[10] Reddy SS, Bijwe PR. Efficiency improvements in meta-heuristic algorithms to solve the optimal power flow problem. Int J Electr Power Energy Syst 2016;82:288-302. http://dx.doi.org/10.1016/j.ijepes.2016.03.028.

[11] Bouchekara HREH, Chaib AE, Abido MA, El-Sehiemy RA. Optimal power flow using an Improved Colliding Bodies Optimization algorithm. Appl Soft Comput 2016;42:119-31. http://dx.doi.org/10.1016/j.asoc.2016.01.041.

[12] Chaib AE, Bouchekara HREH, Mehasni R, Abido MA. Optimal power flow with emission and non-smooth cost functions using backtracking search optimization algorithm. Int J Electr Power Energy Syst 2016;81:64-77. http://dx.doi.org/10. 1016/j.ijepes.2016.02.004

[13] Wei H, Sasaki H, Kubokawa J, Yokoyama R. An interior point nonlinear programming for optimal power flow problems with a novel data structure. IEEE Trans Power Syst 1998;13:870-7. http://dx.doi.org/10.1109/59.708745.

[14] Jabr RA, Coonick AH, Cory BJ. A primal-dual interior point method for optimal power flow dispatching. IEEE Trans Power Syst 2002;17:654-62. http://dx.doi.org/ 10.1109/TPWRS.2002.800870.

[15] Wu Y-C, Debs AS, Marsten RE. A direct nonlinear predictor-corrector primal-dual interior point algorithm for optimal power flows. IEEE Trans Power Syst 1994;9:876-83. http://dx.doi.org/10.1109/59.317660.

[16] Chung CY, Yan W, Liu F. Decomposed predictor-corrector interior point method for dynamic optimal power flow. Power Syst IEEE Trans 2011;26:1030-9. http://dx. doi.org/10.1109/TPWRS.2010.2080326.

[17] Sousa AA, Torres GL, Canizares CA. Robust optimal power flow solution using trust region and interior-point methods. IEEE Trans Power Syst 2011;26:487-99. http:// dx.doi.org/10.1109/TPWRS.2010.2068568.

[18] Duan C, Fang W, Jiang L, Liu J. Adaptive barrier filter-line-search interior point method for optimal power flow with FACTS devices. IET Gener Transm Distrib 2015;9:2792-8. http://dx.doi.org/10.1049/iet-gtd.2015.0623.

[19] Papageorgiou LG, Fraga ES. A mixed integer quadratic programming formulation for the economic dispatch of generators with prohibited operating zones. Electr Power Syst Res 2007;77:1292-6. http://dx.doi.org/10.1016/j.epsr.2006.09.020.

[20] Ding T, Bo R, Gu W, Sun H. Big-M based MIQP method for economic dispatch with disjoint prohibited zones. Power Syst IEEE Trans 2014;29:976-7. http://dx.doi.org/ 10.1109/TPWRS.2013.2287993.

[21] Pourakbari-Kasmaei M, Rider MJ, Mantovani JRS. An unambiguous distance-based
MIQP model to solve economic dispatch problems with disjoint operating zones. Power Syst IEEE Trans 2016;31:825-6. http://dx.doi.org/10.1109/TPWRS.2015. 2394317.

[22] Jabr RA. Solution to economic dispatching with disjoint feasible regions via semidefinite programming. Power Syst IEEE Trans 2012;27:572-3. http://dx.doi.org/ 10.1109/TPWRS.2011.2166009.

[23] Mohammadi-Ivatloo B, Rabiee A, Soroudi A. Nonconvex dynamic economic power dispatch problems solution using hybrid immune-genetic algorithm. IEEE Syst J 2013;7:777-85. http://dx.doi.org/10.1109/JSYST.2013.2258747.

[24] Akbari T, Bina MT. Linear approximated formulation of AC optimal power flow using binary discretisation. IET Gener Transm Distrib 2016;10:1117-23. http://dx. doi.org/10.1049/iet-gtd.2015.0388.

[25] Yang Z, Zhong H, Xia Q, Bose A, Kang C. Optimal power flow based on successive linear approximation of power flow equations. IET Gener Transm Distrib 2016;10:3654-62. http://dx.doi.org/10.1049/iet-gtd.2016.0547.

[26] Fourer R, Gay DM, Kernighan BW. AMPL: a modeling language for mathematical programming. Duxbury Press; 2002.

[27] The GAMS Development Corporation Website. Available at: http://www.gams.com; 2010.

[28] Decker GL, Brooks AD. Valve point loading of turbines. Electr Eng 1958;77:501. http://dx.doi.org/10.1109/EE.1958.6445133.

[29] Rahmani M, Rashidinejad M, Carreno E, Romero RA. Efficient method for AC transmission network expansion planning. Electr Power Syst Res 2010;80:1056-64. http://dx.doi.org/10.1016/j.epsr.2010.01.012.

[30] Pourakbari-Kasmaei M, Contreras J, Mantovani JRS. A demand power factor-based approach for finding the maximum loading point. Electr Power Syst Res 2017;151:283-95. http://dx.doi.org/10.1016/j.epsr.2017.05.042.

[31] Basu M. Improved differential evolution for economic dispatch. Int J Electr Power Energy Syst 2014;63:855-61. http://dx.doi.org/10.1016/j.ijepes.2014.07.003.

[32] Pourakbari-Kasmaei M, Rider MJ, Mantovani JRS. An unequivocal normalizationbased paradigm to solve dynamic economic and emission active-reactive OPF (optimal power flow). Energy 2014;73:554-66. http://dx.doi.org/10.1016/j. energy.2014.06.057.

[33] Monticelli AJ. Fluxo de Carga em Redes de Energia Elétrica. CEPEL; 1983.

[34] AlRashidi MR, El-Hawary ME. Hybrid particle swarm optimization approach for solving the discrete OPF problem considering the valve loading effects. Power Syst IEEE Trans 2007;22:2030-8. http://dx.doi.org/10.1109/TPWRS.2007.907375.

[35] Sivasubramani S, Swarup KS. Sequential quadratic programming based differential evolution algorithm for optimal power flow problem. Gener Transm Distrib IET 2011;5:1149-54. http://dx.doi.org/10.1049/iet-gtd.2011.0046.

[36] Basu M. Multi-objective optimal power flow with FACTS devices. Energy Convers Manag 2011;52:903-10. http://dx.doi.org/10.1016/j.enconman.2010.08.017.

[37] Byrd R, Nocedal J, Waltz R. Knitro: an integrated package for nonlinear optimization. In: Di Pillo G, Roma M, editors. Large-scale nonlinear optim. US: Springer; 2006. p. 35-59. http://dx.doi.org/10.1007/0-387-30065-1_4.

[38] Dieu VN, Ongsakul W. Ramp rate constrained unit commitment by improved priority list and augmented Lagrange Hopfield network. Electr Power Syst Res 2008;78:291-301. http://dx.doi.org/10.1016/j.epsr.2007.02.011.

[39] Online, Available: http://www.ee.washington.edu/research/pstca.

[40] Niknam T, Narimani MR, Azizipanah-Abarghooee R. A new hybrid algorithm fo optimal power flow considering prohibited zones and valve point effect. Energy Convers Manag 2012;58:197-206. http://dx.doi.org/10.1016/j.enconman.2012 01.017.

[41] Sayah S, Zehar K. Modified differential evolution algorithm for optimal power flow with non-smooth cost functions. Energy Convers Manag 2008;49:3036-42. http:// dx.doi.org/10.1016/j.enconman.2008.06.014.

[42] Reddy SS, Bijwe PR, Abhyankar AR. Faster evolutionary algorithm based optimal power flow using incremental variables. Int $\mathrm{J}$ Electr Power Energy Syst 2014;54:198-210. http://dx.doi.org/10.1016/j.ijepes.2013.07.019.

[43] Kumari MS, Maheswarapu S. Enhanced genetic algorithm based computation technique for multi-objective optimal power flow solution. Int J Electr Power Energy Syst 2010;32:736-42. http://dx.doi.org/10.1016/j.ijepes.2010.01.010.

[44] Zimmerman RD, Murillo-Sanchez CE, D.G., MATPOWER, [Online]. Available: http://www.pserc.cornell.edu/matpower/. 